Pacific Journal of Mathematics

ON HIGH SUBGROUPS

charles Kimbrough Megibben, III 


\title{
ON HIGH SUBGROUPS
}

\author{
Charles K. Megibben
}

One of the purposes of this paper is to answer the following three questions:

(1) What groups $G$ with $G^{1}=0$ are direct summands of all groups containing them as high subgroups?

(2) If $G$ is a $\Sigma$-group, are all high subgroups of $G$ endomorphic images of $G$ (see [3] and [4])?

(3) If $G$ is a torsion $\Sigma$-group, is every subgroup of $G$ a $\Sigma$-group (see [3])?

The answer is affirmative to (2) and negative to (3). However an affirmative answer can be given to (3) when $\left|G^{1}\right| \leqq \aleph_{0}$.

All groups in this paper will be assumed to be additively written abelian groups. For the most part, the notation and terminology of [2] will be followed. If $G$ is a group, $G_{t}$ will denote the torsion subgroup of $G$ and $G^{1}$ the subgroup of elements of infinite height, that is, $G^{1}=\bigcap_{n=1}^{\infty} n G$. A torsion group is said to be closed if each $p$-primary component is a closed p-group (see [2], pp. 114-117). A mixed abelian group is said to split if it decomposes into a direct sum of a torsion and torsion free group. By the $n$-adic topology on the group $G$, we shall mean the topology defined by taking as neighborhoods of 0 the subgroups $n G$ for each positive integer $n$. A subgroup $H$ of $G$ is said to be a high subgroup if $H$ is maximal in $G$ with respect to $H \cap G^{1}=0$. If $H$ is a high subgroup of $G$, then $H$ is pure in $G$ and $G / H$ is divisible (see [3]). If all high subgroups of $G$ are direct sums of cyclic groups, then $G$ is said to be a $\Sigma$-group. If one high subgroup of $G$ is a direct sum of cyclic groups, then all high subgroups of $G$ are isomorphic and $G$ is a $\Sigma$-group (see [4]).

1. High subgroups. Let $G$ be an arbitrary abelian group and let $D$ be a minimal divisible group containing $G^{1}$. Then let $K$ be the amalgamated sum of $G$ and $D$ over $G^{1}$, that is, $K$ is the abelian group generated by the elements of $G$ and $D$ subject only to $G \cap D=G^{1}$. ( $K$ can be realized as $(G+D) / L$ where $L$ is the subgroup of $G+D$ consisting of all elements of the form $(x,-x)$ with $x \in G^{1}$.) It then follows that $K / G=\{G, D\} / G \cong D /(G \cap D)=D / G^{1}$, and similarly that $K / D \cong G / G^{1}$.

Lemma 1. If $D$ is minimal divisible containing $G^{1}$ and if $K$ is the amalgamated sum of $G$ and $D$ over $G^{1}$, then

Received September 26, 1963, and in revised form December 3, 1963. 
(i) $G$ is a pure subgroup of $K$;

(ii) $K=H+D$ with $H \cong G / G^{1}$;

(iii) $H \cap G$ is a high subgroup of $G$;

(iv) $K=\{H, G\}$; and

(v) $G$ is a subdirect sum of $H$ and $D$.

Proof. If $g \in G$ and $n k=g$ for some $k \in K$, then we write $k=$ $g_{1}+d$ with $g_{1} \in G$ and $d \in D$. Then $n d=g-n g_{1}$ is an element of $G \cap D=G^{1}$ and hence there is a $g_{2} \in G$ such that $n g_{2}=g-n g_{1}$, that is, $g=n\left(g_{1}+g_{2}\right)$ and we conclude that $G$ is pure in $K$.

(ii) is immediate since divisible subgroups are always direct summands and, as observed above, $K / D \cong G / G^{1}$.

In order to show that $H \cap G$ is high in $G$ we need only prove for every $g \in G \backslash H$ that $\{H \cap G, g\} \cap G^{1} \neq 0$. If $g \in G \backslash H$, we write $g=$ $h+d$ with $h \in H, d \in D$ and $d \neq 0$. Since $D$ is minimal divisible containing $G^{1}$, for some integer $n, n d$ is a nonzero element in $G^{1}$. Then $n h=n(g-d) \in H \cap G$ and $n d=n g-n h$ is a nonzero element of $\{H \cap G, g\} \cap G^{1}$.

Let $p$ be an arbitrary prime. Since $G^{1}[p]=D[p], K[p] \subseteq\{H, G\}$. Assume that we have established that $K\left[p^{n}\right]$ is contained in $\{H, G\}$. In order to show that $K\left[p^{n+1}\right]$ is contained in $\{H, G\}$, we need only consider elements in $D\left[p^{n+1}\right]$. If $d \in D\left[p^{n+1}\right]$, then $p^{n} d \in G^{1}$ and therefore there is a $g \in G$ such that $d-g \in K\left[p^{n}\right] \subseteq\{H, G\}$, that is, $d \in\{H, G\}$ and we conclude that $K\left[p^{n+1}\right] \leqq\{H, G\}$. Clearly then the torsion subgroup of $K$ is contained in $\{H, G\}$. To complete the proof that $K=\{H, G\}$, we show that $D \cong\{H, G\}$. Indeed if $d \in D$ with $d \neq 0$, then $n d$ is a nonzero element of $G^{1}$ for some integer $n$. Then there is a $g \in G$ such that $d-g \in K[n] \subseteq\{H, G\}$ and therefore $d \in\{H, G\}$.

Since $K=\{H, G\}=\{D, G\}$, for each $h \in H$ there is a $g \in G$ and a $d \in D$ such that $g=h+d$; and similarly, for each $d \in D$ there is a $g \in G$ and an $h \in H$ such that $g=h+d$. Thus, $G$ is a subdirect sum of $H$ and $D$ and the kernels are obviously $H \cap G$ and $D \cap G=G^{1}$.

Lemma 1 suggests a useful method for constructing groups with certain properties. Indeed, it can be shown without difficulty that if $D$ is minimal divisible containing the group $A$ and if $H$ is a group without elements of infinite height having a pure subgroup $B$ such that $H / B \cong D / A$, then any subdirect sum $G$ of $H$ and $D$ with kernels $B$ and $A$ is a pure subgroup of $H+D$ such that

(i) $G^{1}=A$,

(ii) $G / G^{1} \cong H$, and

(iii) $B$ is a high group of $G$.

THEOREM 1. Let $M$ be an abelian group without elements of 
infinite height. Then $M$ is a direct summand of every group containing it as a high subgroup if and only if $M_{t}$ is closed.

Proof. Suppose that $M_{t}$ is closed and that $G$ contains $M$ as a high subgroup with $G / M=D$. The $G$ can be represented as a subdirect sum of $H$ and $D$ where $H \cong G / G^{1}$ and $M=H \cap G$. Therefore $D / G^{1} \cong$ $H / M \cong\left(H / M_{t}\right) /\left(M / M_{t}\right)$ and since $M / M_{t}$ is a torsion free pure subgroup of $H / M_{t}$ and $D / G^{1}$ is torsion, $H / M_{t}=J / M_{t}+M / M_{t}$. But since $M_{t}$ is closed and is pure in the torsion group $J, J=L+M_{t}$ with $L \cong D / G^{1}$. Thus $H=L+M$ and since $M$ is a direct summand of $H, M$ is necessarily a direct summand of $G$.

Suppose new that $M_{t}$ is not closed. Let $M^{*}$ be the $n$-adic completion of $M$. Then $M$ is a pure subgroup of $M^{*}$ with $M^{*} / M$ divisible. Since $M_{t}^{*}$ is closed, $\left\{M, M_{t}^{*}\right\} / M$ is nonzero and is moreover the torsion subgroup of $M^{*} / M$. Let $H=\left\{M, M_{t}^{*}\right\}$ and choose a direct sum $A$ of cyclic groups such that if $D$ is minimal divisible containing $A$, then $D / A \cong$ $H / M$. If $G$ is a subdirect sum of $H$ and $D$ with kernels $M$ and $A$, then $G$ will be a reduced group having $M$ as a high subgroup.

THEOREM 2. If some high subgroup of $G$ splits, then $G / G^{1}$ splits.

Proof. Let $T+F$ be a high subgroup of $G$ where $T$ is torsion and $F$ is torsion free. Let $D$ be minimal divisible containing $G^{1}$ and let $K$ be the amalgamated sum of $G$ and $D$ over $G^{1}$. Then if $K=$ $H+D$, we may assume that $G \cap H=T+F$. Then $H /(T+F)=$ $H /(H \cap G) \cong\{H, G\} / G=K / G \cong D / G^{1}$. Therefore since $D / G^{1}$ is torsion and $(T+F) / T$ is a torsion free pure subgroup of $H / T, H / T=M / T+$ $(T+F) / T$ for some subgroup $M$ of $H$. Hence $H=M+F$ where $M$ is necessarily a torsion group since $M / T$ is torsion, that is, $H$ splits and $H \cong G / G^{1}$.

COROLlaRY 1. If some high subgroup of $G$ is torsion, then $G / G^{1}$ is torsion and therefore all high subgroups of $G$ are torsion.

Corollary 2. (Irwin, Peercy and Walker [4]) If $A$ is a high subgroup of $G$ and $A=T+F$ where $T$ is torsion and $F$ is torsion free, then $G=L+F$ with $L / T$ divisible.

Proof. Let $D$ and $K$ be as in the proof of Theorem 2 and suppose that $K=H+D$ with $H \cap G=A$. Then $K=(M+F)+D$ and therefore $G=L+F$ where $L=G \cap(M+D)$. Finally, we observe that $L / T \cong G /(T+F)=G /(H \cap G) \cong D$.

REMARK. From an example in [4], $G$ need not split if $G / G^{1}$ splits. 


\section{2. $\Sigma$-groups.}

THEOREM 3. If $G$ is a $\Sigma$-group, then every high subgroup of $G$ is an endomorphic image of $G$. More generally, if the high subgroup $H$ of $G$ splits and the torsion subgroup of $H$ is a direct sum of cyclic groups, then $H$ is an endomorphic image of $G$.

Proof. If $H=T+F$ where $T$ is torsion and $F$ is torsion free, then $G / G^{1} \cong M+F$ where $M$ is torsion and contains $T$ as a pure subgroup. If $T$ is a direct sum of cyclic groups, then $T$ is a basic subgroup of $M$ and therefore an endomorphic image of $M$. Clearly then if $T$ is a direct sum of cyclic groups, $H$ is an endomorphic image of $G$.

Requiring a $\Sigma$-group to have at most countably many elements of infinite height imposes severe restrictions on the structure of the group.

THEOREM 4. If $G$ is a $\Sigma$-group such that $\left|G^{1}\right| \leqq \aleph_{0}$, then $G / G^{1}$ is a direct sum of cyclic groups.

Proof. If $H=T+F$ is a high subgroup of $G$ and if $F$ is free and $T$ is a torsion direct sum of cyclic groups, then $G / G^{1} \cong M+F$ where $M$ is a torsion direct sum of cyclic groups provided $\left|G^{1}\right| \leqq \aleph_{0}$. Indeed, $M / T \cong D / G^{1}$, where $D$ is minimal divisible containing $G^{1}$, and since $D / G^{1}$ is necessarily at most countable and $M$ is without elements of infinite height, Theorem 33.4 in [2] implies that $M$ is a direct sum of cyclic groups.

REMARK. A glance at the proof of Theorem 4 should suggest an extremely simple proof of the fact that if one high subgroup of a group is a direct sum of cyclic groups then all high subgroups of the group are isomorphic.

THeOREM 5. If $G$ is a torsion $\Sigma$-group and $G^{1}$ has an at most countable basic subgroup, then $G / G^{\mathrm{r}}$ is a direct sum of cyclic groups.

Proof. We need only observe that if $G^{1}$ has an at most countable basic subgroup and if $D$ is minimal divisible containing $G^{1}$, then $\left|D / G^{1}\right| \leqq \aleph_{0}$ (see [2], p. 110).

EXAmple 1. The restrictions in Theorems 3 and 4 are necessary. Indeed, let $B=\sum_{n=1}^{\infty} C\left(p^{n}\right)$ where $p$ is a prime and let $\bar{B}$ be the torsion subgroup of $\sum_{n=1}^{\infty} * C\left(p^{n}\right)$. Then $B$ is pure in $\bar{B}$ and $\bar{B} / B$ is isomorphic to $2^{\aleph_{0}}$ copies of $C\left(p^{\infty}\right)$. Next set $A=\sum_{\lambda \in A}\left\{a_{\lambda}\right\}$, where $\left\{a_{\lambda}\right\} \cong C(p)$ for each $\lambda$ and $|A|=2^{\aleph_{0}}$. Then if $D$ is minimal divisible containing: 
$A, D / A \cong \bar{B} / B$. If $G$ is a subdirect sum of $\bar{B}$ and $D$ with kernels $\bar{B}$ and $A$, then $G$ is a $\Sigma$-group such that $G / G^{1} \cong \bar{B}$.

The proof of the following lemma follows immediately from results in $[1]$.

LEMma 2. If $A$ is an at most countable subgroup of the torsion group $G$ such that $G / A$ is a direct sum of cyclic groups, then $G$ is the direct sum of an at most countable group and a direct sum of cyclic groups.

THEOREM 6. If $G$ is a torsion group such that $\left|G^{1}\right| \leqq \aleph_{0}$, then the following three conditions are equivalent:

(i) $G$ is a $\Sigma$-group.

(ii) $G / G^{1}$ is a direct sum of cyclic groups.

(iii) $G=H+C$ where $|H| \leqq \aleph_{0}$ and $C$ is a direct sum of cyclic groups.

Proof. (i) implies (ii) by Theorem 4. (ii) implies (iii) by Lemma 2. And, finally, it is easy to see that (iii) always implies (i).

THEOREM 7. If $G$ is a torsion $\Sigma$-group such that $\left|G^{1}\right| \leqq \aleph_{0}$, then every subgroup of $G$ is a $\Sigma$-group.

Proof. Let $G$ be a torsion $\Sigma$-group such that $\left|G^{1}\right| \leqq \aleph_{0}$ and let $H$ be a subgroup of $G$. In order to show that $H$ is a $\Sigma$-group, it suffices to show that $H / H^{1}$ is a direct sum of cyclic groups. Since $H / H \cap G^{1} \cong\left\{H, G^{1}\right\} / G^{1}, H / H \cap G^{1}$ is a direct sum of cyclic groups. But this group is isomorphic to $\left(H / H^{1}\right) /\left(H \cap G^{1} / H^{1}\right)$ and since $H \cap G^{1} / H^{1}$ is at most countable and $H / H^{1}$ is without elements of infinite height, we conclude from Lemma 2 that $H / H^{1}$ is a direct sum of cyclic groups.

COROLLARY 3. If the torsion group $G$ is the direct sum of a countable group and a direct sum of cyclic groups, then every subgroup of $G$ has a similar direct decomposition.

Example 2. The restriction that $\left|G^{1}\right| \leqq \aleph_{0}$ in Theorem 7 is necessary. Indeed, if $\bar{B}$ is as in Example 1 , it is then easy to construct by methods we have used above a primary $\Sigma$-group $G$ with $G^{1}=\bar{B}$. Then $G^{1}$ is itself a subgroup of $G$ which is not a $\Sigma$-group.

\section{REFERENCES}

1. B. Charles, Note sur la structure des groupes abéliens primaires, C. R. Acad. Sci. Paris, 252 (1961), 1547-1548. 
2. L. Fuchs, Abelian groups, Budapest, 1958.

3. J. Irwin and E. Walker, On N-high subgroups of abelian groups, Pacific J. Math., 11 (1960), 1363-1374.

4. J. Irwin, C. Peercy and E. Walker, Splitting properties of high subgroups, Bull. Soc. Math. France, 90 (1962), 185-192.

Texas Technologial College, Lubbock 


\title{
PACIFIC JOURNAL OF MATHEMATICS
}

\author{
EDITORS
}

\author{
RoBerit OsSERman \\ Stanford University \\ Stanford, California
University of Washington
Seattle 5 , Washington \\ M. G. Arsove
}

\author{
J. DugundjI \\ University of Southern California
}

Los Angeles 7, California

Lowell J. Paige

University of California

Los Angeles 24, California

\section{ASSOCIATE EDITORS}
E. F. BECKENBACH
B. H. NeumanN
F. WOLF
K. YOSIDA

\section{SUPPORTING INSTITUTIONS}

\author{
UNIVERSITY OF BRITISH COLUMBIA \\ CALIFORNIA INSTITUTE OF TECHNOLOGY \\ UNIVERSITY OF CALIFORNIA \\ MONTANA STATE UNIVERSITY \\ UNIVERSITY OF NEVADA \\ NEW MEXICO STATE UNIVERSITY \\ OREGON STATE UNIVERSITY \\ UNIVERSITY OF OREGON \\ OSAKA UNIVERSITY \\ UNIVERSITY OF SOUTHERN CALIFORNIA
}

\author{
STANFORD UNIVERSITY \\ UNIVERSITY OF TOKYO \\ UNIVERSITY OF UTAH \\ WASHINGTON STATE UNIVERSITY \\ UNIVERSITY OF WASHINGTON \\ * * * * \\ AMERICAN MATHEMATICAL SOCIETY \\ CALIFORNIA RESEARCH CORPORATION \\ SPACE TECHNOLOGY LABORATORIES \\ NAVAL ORDNANCE TEST STATION
}

Mathematical papers intended for publication in the Pacific Journal of Mathematics should by typewritten (double spaced), and on submission, must be accompanied by a separate author's résumé. Manuscripts may be sent to any one of the four editors. All other communications to the editors should be addressed to the managing editor, L. J. Paige at the University of California, Los Angeles 24, California.

50 reprints per author of each article are furnished free of charge; additional copies may be obtained at cost in multiples of 50 .

The Pacific Journal of Mathematics is published quarterly, in March, June, September, and December. Effective with Volume 13 the price per volume (4 numbers) is $\$ 18.00$; single issues, $\$ 5.00$. Special price for current issues to individual faculty members of supporting institutions and to individual members of the American Mathematical Society: $\$ 8.00$ per volume; single issues $\$ 2.50$. Back numbers are available.

Subscriptions, orders for back numbers, and changes of address should be sent to Pacific Journal of Mathematics, 103 Highland Boulevard, Berkeley 8, California.

Printed at Kokusai Bunken Insatsusha (International Academic Printing Co., Ltd.), No. 6, 2-chome, Fujimi-cho, Chiyoda-ku, Tokyo, Japan.

PUBLISHED BY PACIFIC JOURNAL OF MATHEMATICS, A NON-PROFIT CORPORATION

The Supporting Institutions listed above contribute to the cost of publication of this Journal. but they are not owners or publishers and have no responsibility for its content or policies. 


\section{Pacific Journal of Mathematics}

\section{Vol. 14, No. $4 \quad$ August, 1964}

Homer Franklin Bechtell, Jr., Pseudo-Frattini subgroups . . . . . . . . . . . . . . . . 1129

Thomas Kelman Boehme and Andrew Michael Bruckner, Functions with convex means .............................................. 1137

Lutz Bungart, Boundary kernel functions for domains on complex manifolds . . . . . 1151

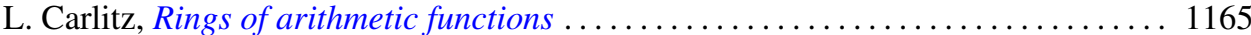

D. S. Carter, Uniqueness of a class of steady plane gravity flows . . . . . . . . . 1173

Richard Albert Dean and Robert Harvey Oehmke, Idempotent semigroups with

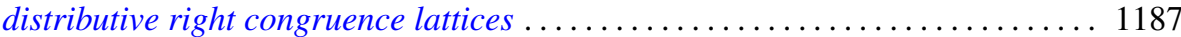

Lester Eli Dubins and David Amiel Freedman, Measurable sets of measures ..... . 1211

Robert Pertsch Gilbert, On class of elliptic partial differential equations in four

variables.......................................... 1223

Harry Gonshor, On abstract affine near-rings .................... 1237

Edward Everett Grace, Cut points in totally non-semi-locally-connected

continua ........................................... 1241

Edward Everett Grace, On local properties and $G_{\delta}$ sets ................ 1245

Keith A. Hardie, A proof of the Nakaoka-Toda formula . . . . . . . . . . . . . . . . 1249

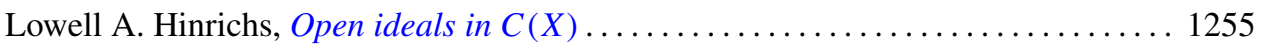

John Rolfe Isbell, Natural sums and abelianizing . . . . . . . . . . . . . . . . 1265

G. W. Kimble, A characterization of extremals for general multiple integral

problems............................................. 1283

Nand Kishore, A representation of the Bernoulli number $B_{n} \ldots \ldots \ldots \ldots \ldots \ldots \ldots \ldots$

Melven Robert Krom, A decision procedure for a class of formulas of first order predicate calculus.

Peter A. Lappan, Identity and uniqueness theorems for automorphic functions ...

Lorraine Doris Lavallee, Mosaics of metric continua and of quasi-Peano spaces . .

Mark Mahowald, On the normal bundle of a manifold..........

J. D. McKnight, Kleene quotient theorems

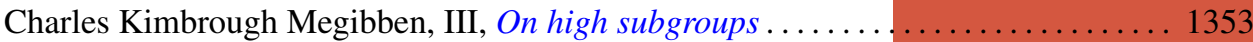

Philip Miles, Derivations on $B^{*}$ algebras . . . . . . . . . . . . . . . . . . . 1359

J. Marshall Osborn, A generalization of power-associativity . . . . . . . . . . . 1367

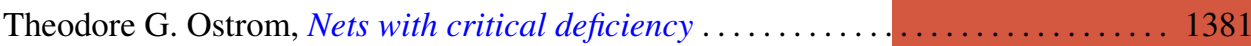

Elvira Rapaport Strasser, On the defining relations of a free product . . . . . . . . . 1389

K. Rogers, A note on orthoganal Latin squares . . . . . . . . . . . . . . . . . . 1395

P. P. Saworotnow, On continuity of multiplication in a complemented algebra ..... 1399

Johanan Schonheim, On coverings . . . . . . . . . . . . . . . . . . . . 1405

Victor Lenard Shapiro, Bounded generalized analytic functions on the torus . . . . . 1413

James D. Stafney, Arens multiplication and convolution . . . . . . . . . . . . . 1423

Daniel Sterling, Coverings of algebraic groups and Lie algebras of classical

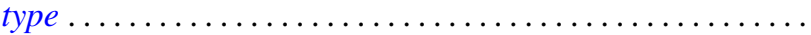

Alfred B. Willcox, Šlov type $C$ algebras over a connected locally compact abelian

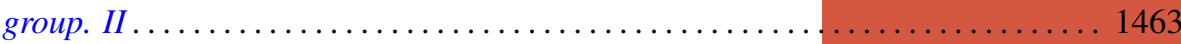

Bertram Yood, Faithful ${ }^{*}$-representations of normed algebras. II . . . . . . . . . 1475

Alexander Zabrodsky, Covering spaces of paracompact spaces 